\section{Guidelines for the management of bacterial and fungal infections during chemotherapy for pediatric acute leukemia or solid tumors: what is available in 2010?}

\author{
Elio Castagnola, 1 Ilaria Caviglia, 1 \\ Riccardo Haupt?
}

IInfectious Diseases Unit, Oncohematology Department, G. Gaslini Children's Hospital, Genoa; 2Epidemiology and Biostatistics Section, Scientific Directorate, G. Gaslini Children's Hospital, Genoa, Italy

\section{Abstract}

Febrile episodes and infections represent important complications during antineoplastic chemotherapy for pediatric neoplastic diseases. In the last years many international association published guidelines for the management of these complications in adults, but no document of this type was prepared for children. One of the major causes of this situation is probably the very low number of pediatric clinical trials with adequate power and design. The paper summarizes guidelines provided for the management of infectious complications in adults with cancer by different international and will comment on how much they may be translated in the management of pediatric patients.

\section{Introduction}

Febrile episodes and infections represent important complications during antineoplastic chemotherapy for pediatric neoplastic diseases. In the last years many international association published guidelines for the management of these complications in adults, but no document of this type was prepared for children. One of the major causes of this situation is probably the very low number of pediatric clinical trials with adequate power and design: for example there is only one double blind placebo-controlled trial of antibacterial prophylaxis of febrile neutropenia, published in 2003 and including 173 patients, ${ }^{1}$ while the first study on empirical antifungal therapy for persistent febrile neutropenia in children was published in 2010, and included 163 subjects. ${ }^{2}$ As a consequence, pediatric hematologist and oncologist frequently apply guidelines implemented for adults even if children present dif- ferences as regards pharmacology of drugs, epidemiology and, sometimes, clinical features of infectious complications during antineoplastic chemotherapy.

In the following paragraphs we will summarize guidelines provided for the management of infectious complications in adults with cancer by different international Societies, i.e. the European Conference for Infections in Leukaemia (ECIL) (available at http://www. ichs.org), Infectious Diseases Society of America (IDSA), ${ }^{3}$ ) British Committee for Standards in Haematology (BCSH), ${ }^{4}$ German Society for Hematology and Oncology (DGHO), ${ }^{5-7}$ National Comprehensive Cancer Network (NCCN, available at http://www. nccn.org), and will comment on how much they may be translated in the management of pediatric patients, mainly using as a base an epidemiological and clinical "common sense", more than the availability of adequate pediatric studies. It must be noted that only the NCCN guideline includes patients with solid tumors while all the others are referred to patients with acute leukemia, lymphoma or myelodisplastic syndrome.

\section{Prophylaxis}

In any type of clinical condition the decision of administering anti-infective prophylaxis must generally be based on the answers to 4 major questions: i) could you easily treat the event you are trying to prevent if it happened? (No: prophylaxis, Yes: no prophylaxis); ii) is it a severe event? (Yes: prophylaxis, No: no prophylaxis); iii) does the prophylaxis have adverse effects, including development of resistance? (No: prophylaxis, Yes: no prophylaxis); iv) is the prophylaxis effective, i.e. which is the number of patients needed to treat (NNT) to prevent one event? (low: prophylaxis, high: no prophylaxis). ${ }^{8}$ The NNT is calculated with the formula $1 /$ absolute reduction of the risk (i.e. ratio in the experimental group - ratio in controls), but there is no standardization to decide if the NNT is satisfactory, since it could depend on frequency of the disease in the controls, consequences of the treatments (efficacy vs. toxicity and/or drug interactions), costs (of prophylaxis and the treatment of the disease if not prevented), selection of resistances. ${ }^{9}$

Table 1 summarizes the recommendations reported in different guidelines for antibacterial and antifungal prophylaxis in adults receiving antineoplastic chemotherapy.

As regards the pediatric population the efficacy of antibacterial prophylaxis for the prevention of febrile neutropenia was evaluated in only one randomized, double blind, placebocontrolled trial, ${ }^{1}$ which showed a statistically
Correspondence: Elio Castagnola, Infectious Diseases Unit, Oncohematology Department, G. Gaslini Children Hospital, Largo G. Gaslini, 5 16147, Genoa, Italy.

Tel. +39.010.5636.428 - Fax. +39.010.376.34.36

E-mail: eliocastagnola@ospedale-gaslini.ge.it

Key words: pediatric leukemia, pediatric solid tumor, bacterial and fungal infections.

Received for publication: 16 November 2010 Accepted for publication: 13 December 2010.

This work is licensed under a Creative Commons Attribution 3.0 License (by-nc 3.0).

(C) Copyright E. Castagnola et al., 2011

Licensee PAGEPress, Italy

Pediatric Reports 2011; 3:e7

doi:10.4081/pr.2011.e7

significant protective effect in children with acute leukemia/ymphoma (-17\%), but not in solid tumors, with a NNT of 6 patients. Analyses of studies performed in adults receiving fluoroquinolones showed a NNT of 5 patients to prevent a febrile episode, of 33 to prevent one death and of 23 to prevent an infection-related death. ${ }^{10,11}$ The use of antibacterial prophylaxis (with fluoroquinolones, since we are dealing with adults) is highly recommended in the ECIL and NCCN guidelines, but not in the others (Table 1). However, it must be stressed that no study evaluated the effects of repeated cycles of prophylaxis administered during the whole course of antineoplastic chemotherapy, and that the widespread use of antibacterial prophylaxis induce the development of resistance, circumstance that is becoming one of the major concerns in supportive care in cancer.

As regards antifungal prophylaxis, no satisfactory study evaluated the efficacy and effectiveness of this procedure in children. In adults, two recent studies demonstrated the higher efficacy of oral posaconazole $(-6 \%$ of events $)^{12}$ or nebulized liposomal amphotericin B + fluconazole $(-10 \% \text { of events })^{13}$ compared with other drugs or placebo in reducing proven/probable invasive fungal diseases during repeated periods at risk following chemotherapy for acute leukemia. Posaconazole prophylaxis is therefore highly recommended by ECIL, DGHO and NCCN, while nebulized liposomal amphotericin B + fluconazole has a lower level of recommendation in the ECIL and DGHO guidelines (Table 1). Stemming from these proposal, if the reduction of invasive fungal disease observed in adults was applied to the epidemiological data on invasive fungal diseases observed in pediatrics, ${ }^{14-16}$ the NNT in children would range between 13 and more than 25 , with higher val- 
ues in acute lymphoblastic leukemia. However, some considerations must be done about these two studies. At present the pediatric dosage of posaconazole in almost unknown, since it was evaluated only in 12 children aged 8-17 years, ${ }^{17}$ and many concern have been raised on pharmacokinetics parameters of posaconazole that could be of great importance in pediatrics (need of a fat meal, or at least supplemental food or acid drink, possible need of more refracted doses, avoidance of proton-pump inhibitors or administration through a nasogastic tube). ${ }^{18,19}$ As regards the study with liposomal amphotericin B prophylaxis it must be noted that it required a very long time (more than 5 years) to be completed, and this might have introduced a bias in the evaluation of efficacy. Moreover, the compliance with nebulization systems may be lower in younger children than in adults.

Finally, patients with a history of invasive fungal disease may be at high risk of reactivation when undergoing further chemotherapy. In these patients secondary antifungal prophylaxis is recommended. The role of secondary prophylaxis in preventing relapses has never been studied systematically, even if a longer duration of antifungal therapy before hemopoietic stem cell transplant has been associated with a better outcome, and the recurrence of invasive aspergillosis has been attributed to less than a month of antifungal therapy before transplant and with persistence of radiological abnormalities after treatment. The drug for secondary prophylaxis should be chosen according to the etiology of the infection, the localization, the drugs available and their formulations and risks of interactions with other therapies, especially those for the treatment of the underlying disease (ECIL).

\section{Therapy}

Fever during neutropenia has always been considered a medical emergency and should always be considered as due to infection, unless otherwise proven. The use of empirical antibacterial therapy in febrile neutropenic cancer patients has been demonstrated to significantly improve survival, and it is now considered one of the cornerstones of the supportive care in cancer. In the last years it has been demonstrated that all cancer patients are not the same and therefore different anti-bacterial approaches, oral vs. intravenous, are feasible in different patients' groups, e.g. solid tumors vs. acute leukemias. All the guidelines analyzed indicate the feasibility of front-line intravenous monotherapy with the use of antiPseudomonas beta-lactams (ceftazidime, cefepime or piperacillin-tazobactam or a carbapenem) in high-risk patients or the use of

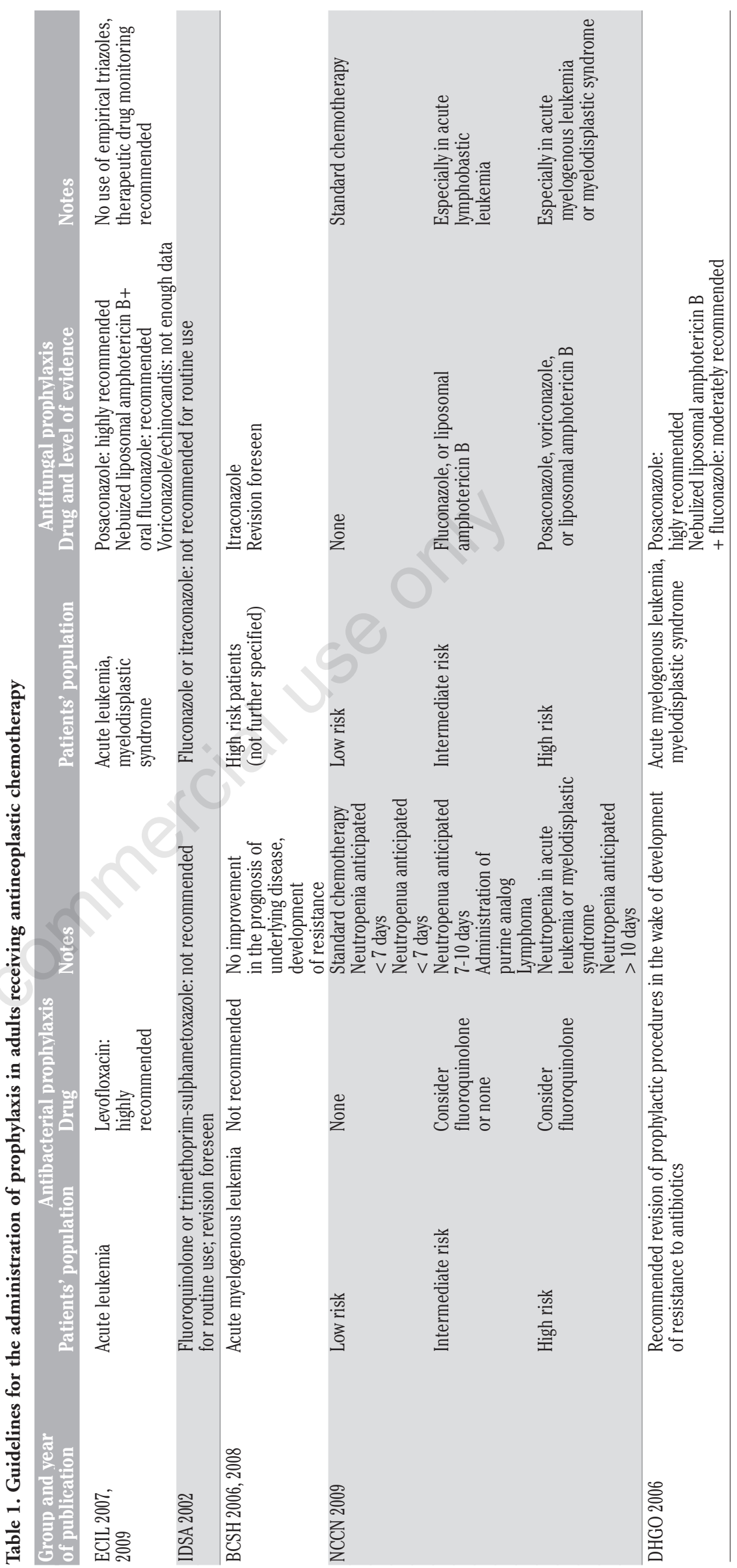


oral therapy with ciprofloxacin + amoxicillinclavulanate in low risk patient's groups (mainly patients with solid tumors). As regards intravenous treatment, the initial use of an aminoglycoside should be limited to the combination with ceftriaxone to give coverage towards Pseudomonas, or in presence of peculiar, local epidemiologic conditions. In case of empirical administration of an aminoglycoside its discontinuation is recommended after 3 days of therapy ( 3 doses) in patients with fever of unknown origin. In all guidelines the empirical use of glycopeptides in initial or in presence of persistent fever is not recommended, unless in presence of microbiological documentation or clinical signs (e.g. skin and soft tissue infection and/or signs of central venous catheter related infection) suggestive of infection due to methicillin-resistant Gram-positives. There are no epidemiological or clinical reasons to suggest a different approach in the pediatric population.

The empirical antifungal therapy has been a common practice in the treatment of persistently febrile neutropenic cancer patients. The rationale for this practice was based on old autopsy studies, showing fungal infections undetected during life and on 2 small, randomized studies, which enrolled less than 200 patients all together. These studies were not double blind or placebo-controlled and actually did not conclude an unequivocal advantage for empirical antifungal therapy. In both studies the statistical power of the observed results was very small, especially for subgroup analyses. ${ }^{20}$ Except the first studies, which used as main endpoint persistence of fever and survival, the most recent studies used a very controversial composite clinical endpoint, which included 5 criteria (defervescence, no discontinuation for toxicity, treatment of baseline fungal infections, prevention of breakthrough fungal infections and survival). Many drugs have been tested for this indication (liposomal amphotericin B, caspofungin, voriconazole, fluconazole), ${ }^{20}$ but only recently a pediatric study was published (liposomal amphotericin B vs. caspofungin). ${ }^{2}$ In general, no drug was significantly more effective than the control one and differences were only based on lower toxicity. Empirical antifungal therapy after 5 days of persistent febrile neutropenia is still recommended by some guidelines (IDSA, NCCN), while in others it is not examined (DGHO), or even discouraged (BCSH). The ECIL guideline recommends this practice with a low level of evidence, but suggests that it should be adopted only in centers where routine CT scan and serum galactomannan antigen detection can not be performed. This recommendation is acceptable also for the pediatric population. In the last years, awareness has grown that the empirical approach has resulted in a tremendous overtreatment of just a symptom (fever). This realization and recent advances in diagnosis are making the practice of empirical therapy more problematic and have encouraged development of a different approach called pre-emptive antifungal therapy, aimed at treating a fungal disease when suggestive but not definitive diagnosis is present. The requirement for initiating preemptive therapy hinges around either a radiological result (lung CT scan) or a microbiological result (Aspergillus galactomannan in serum or BAL fluid, glucan detection in serum, cytological detection of fungal hyphae or positive culture on sputum or BAL fluid). At present the feasibility of the pre-emptive therapy has been evaluated in 2 studies of management strategy22,23 and in one where pre-emptive therapy was randomized vs. empirical therapy, ${ }^{21}$ all performed in adults, while no study is available in pediatrics. These data are not considered sufficient to give a recommendation on the use of this approach to persistently febrile neutropenic patients, neither adults nor children.

Indications for the treatment of documented infections, especially due to resistant pathogens, and/or syndromes due to localized infections are beyond the scope of the present document, but are available in many recent reviews. $6,7,20$

\section{References}

1. Castagnola E, Boni L, Giacchino M, et al. A multicenter, randomized, double blind placebo-controlled trial of amoxicillin/ clavulanate for the prophylaxis of fever and infection in neutropenic children with cancer. Pediatr Infect Dis J 2003;22:35965.

2. Maertens JA, Madero L, Reilly AF, et al. A randomized, double-blind, multicenter study of caspofungin versus liposomal amphotericin B for empiric antifungal therapy in pediatric patients with persistent fever and neutropenia. Pediatr Infect Dis J 2010;29:415-20.

3. Hughes WT, Armstrong D, Bodey GP, et al. 2002 guidelines for the use of antimicrobial agents in neutropenic patients with cancer. Clin Infect Dis 2002;34:730-51.

4. Milligan DW, Grimwade D, Cullis J0, et al. Guidelines on the management of acute myeloid leukaemia in adults. British Committee for Standards in Haematology. Br J Haematol 2006;135:450-74.

5. Cornely OA, Bohme A, Buchheidt D, et al. Primary prophylaxis of invasive fungal infections in patients with hematologic malignancies. Recommendations of the Infectious Diseases Working Party of the German Society for Haematology and Oncology. Haematologica 2009;94:113-22.
6. Bohme A, Ruhnke M, Buchheidt D, et al. Treatment of invasive fungal infections in cancer patients--recommendations of the Infectious Diseases Working Party (AGIHO) of the German Society of Hematology and Oncology (DGHO). Ann Hematol 2009;88:97-110.

7. Neuburger S, Maschmeyer G. Update on management of infections in cancer and stem cell transplant patients. Ann Hematol 2006;85:345-56.

8. McQuay HJ, Moore RA. Using numerical results from systematic reviews in clinical practice. Ann Intern Med 1997;126:71-720.

9. Sinclair JC, Cook RJ, Guyatt GH, et al. When should an effective treatment be used? Derivation of the threshold number needed to treat and the minimum event rate for treatment. J Clin Epidemiol 2001; 54:253-62.

10. Gafter-Gvili A, Fraser A, Paul M, Leibovici L. Meta-analysis: antibiotic prophylaxis reduces mortality in neutropenic patients. Ann Intern Med 2005;142:979-95.

11. Leibovici L, Paul M, Cullen M, et al. Antibiotic prophylaxis in neutropenic patients: new evidence, practical decisions. Cancer 2006;107:1743-51.

12. Cornely OA, Maertens J, Winston DJ, et al. Posaconazole vs. fluconazole or itraconazole prophylaxis in patients with neutropenia. TN Engl J Med 2007;356:348-59.

13. Rijnders BJ, Cornelissen JJ, Slobbe L, et al. Aerosolized liposomal amphotericin B for the prevention of invasive pulmonary aspergillosis during prolonged neutropenia: a randomized, placebo-controlled trial. Clin Infect Dis 2008;46:1401-8.

14. Castagnola E, Caviglia I, Pistorio A, et al. Bloodstream infections and invasive mycoses in children undergoing acute leukaemia treatment: a 13-year experience at a single Italian institution. Eur J Cancer 2005;41:1439-45.

15. Castagnola E, Rossi MR, Cesaro S, et al. Incidence of bacteremias and invasive mycoses in children with acute non-lymphoblastic leukemia: Results from a multicenter Italian study. Pediatr Blood Cancer 2010;55:1103-7.

16. Hale KA, Shaw PJ, Dalla-Pozza L, et al. Epidemiology of paediatric invasive fungal infections and a case-control study of risk factors in acute leukaemia or post stem cell transplant. Br J Haematol 2010;149: 263-72.

17. Krishna G, Sansone-Parsons A, Martinho $\mathrm{M}$, et al. Posaconazole plasma concentrations in juvenile patients with invasive fungal infection. Antimicrob Agents Chemother 2007;51:812-8.

18. Krishna G, Moton A, Ma L, et al. Pharmacokinetics and absorption of posaconazole oral suspension under various gastric con- 
ditions in healthy volunteers. Antimicrob Agents Chemother 2009;53:958-66.

19. Dodds Ashley ES VJ, Krishna G, Vickery D, et al. Pharmacokinetics of posaconazole administered orally or by nasogastric tube in healthy volunteers. Antimicrob Agents Chemother 2009;53:2960-4.

20. Viscoli C, Castagnola E. Prophylaxis and Empirical Therapy of Infection in Cancer Patients. In: Mandell GL, Bennett JE, Dolin
R, eds. Principles and Practice of Infectious Diseases. Philadelphia, Churchill Livingstone Elsevier, 2010; pp:3793-807.

21. Cordonnier C, Pautas C, Maury S, et al. Empirical versus preemptive antifungal therapy for high-risk, febrile, neutropenic patients: a randomized, controlled trial. Clin Infect Dis 2009;48:1042-51.

22. Girmenia C, Micozzi A, Gentile G, et al. Clinically driven diagnostic antifungal approach in neutropenic patients: a prospective feasibility study. J Clin Oncol 2010;28:667-74.

23. Maertens J, Theunissen K, Verhoef G, et al. Galactomannan and computed tomography-based preemptive antifungal therapy in neutropenic patients at high risk for invasive fungal infection: a prospective feasibility study. Clin Infect Dis 2005;41: 1242-50. 